\title{
ACCEPTABILITY OF DIFFERENT LIPID-BASED NUTRIENT SUPPLEMENTS FOR ADULTS WITH HIV
}

Hebie $M^{1}$, Jungjohann $S^{2}$, Praygod $G^{3}$ and $S$ Filteau ${ }^{1 *}$

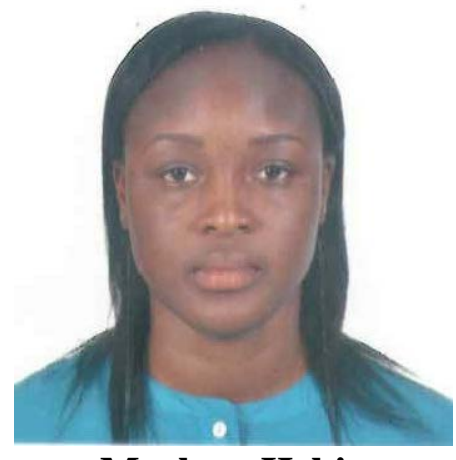

Marlene Hebie

*Corresponding author email: Suzanne.Filteau@lshtm.ac.uk

${ }^{1}$ London School of Hygiene and Tropical Medicine, Keppel Street, London, UK

${ }^{2}$ Nutriset, B.P. 35 - 76770 Malaunay, France

${ }^{3}$ National Institute of Medical Research, Mwanza, Tanzania 


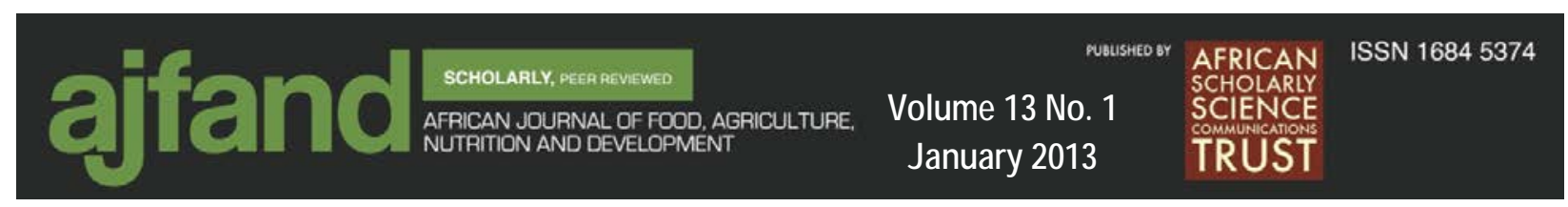

\section{ABSTRACT}

Despite the increased access to antiretroviral therapy, undernutrition is associated with an increased risk of early mortality among HIV-infected adults living in resource-limited settings. Lipid-based Nutrient Supplements (LNS) can provide nutritional support and can be adapted to the needs of adults infected with HIV. However, consumption of these products may be limited by monotony or by an unacceptably strong taste of highly concentrated micronutrients. This study investigated the acceptability of several new flavours of LNS for HIV-infected adults on antiretroviral therapy (ART). Forty HIV-infected adults on ART completed acceptability tests with one of four sets of LNS products: LNS-30 g sweet, LNS-30 g non-sweet, LNS-250 g sweet and LNS-250 g non-sweet. Each set was composed of three flavours and was tasted with and without food during a single session of sensory evaluation. The supplements were block-randomised and the patients were blinded to the type of product. LNS acceptability was assessed based on descriptive, hedonic and food-action rating tests using 5-point rating scales as well as preference tests. All the flavours got a median rate of 3-Just about right for the intensity of sugar, salt, oil, thickness and 4-Good for the taste. Regarding the overall liking, the supplements were positively evaluated with median of 4-I like a little or 5-I like a lot. The LNS-30 g non-sweet formulation was significantly less appreciated when tasted without food compared to all the other sets of LNS $(P=0.0005)$. Mixing LNS with local food had no effect on the acceptability. In terms of consumption pattern, all the products got a median rate of 1-Will eat it at all opportunity or 2-Will eat it very often. Finally, in terms of preference there was no significant difference between the flavours. In LNS$30 \mathrm{~g}$ the overall liking was positively correlated with the taste and the intensity of sugar but the prospective consumption factor was negatively correlated with thickness. In LNS-250 g, only the intensity of salty taste was correlated with the overall liking. As the flavours were well accepted, all of them can be used, providing choice and variety to HIV-infected adults consuming them for prolonged periods. There is no need to modify the products' formulation. Further research on the acceptability of these supplements over the longer term and in settings where food insecurity and dietary patterns are different is warranted.

Key words: LNS, HIV, Acceptability, Sensory trial, Tanzania 


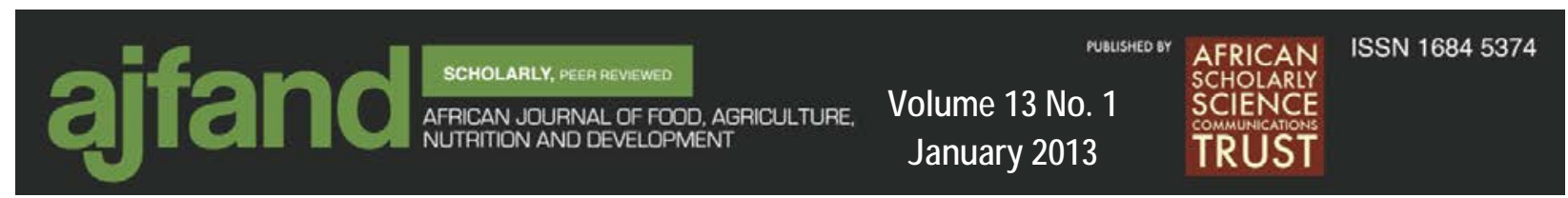

\section{INTRODUCTION}

Human immunodeficiency virus (HIV) infection and undernutrition are concomitant major public health issues in sub-Saharan Africa. According to the 2008 Demographic and Health survey [1], adult HIV prevalence was estimated at $5.7 \%$ in the United Republic of Tanzania, with prevalence over $15 \%$ in some regions. Despite increasing access to antiretroviral therapy (ART), a high early mortality rate has been found among patients in resource-limited settings and multivariate analysis showed that undernutrition was among the leading causes [2-3]. In addition, in both developed and developing countries, low body mass index (BMI) is a strong and independent predictor of morbidity and mortality among HIV-infected individuals regardless of their ART status [3-8].

The World Health Organisation (WHO) recommends for HIV-infected adults an energy intake above the requirements of healthy and normally active HIV-uninfected persons of the same age and sex [9]. Supplementary feeding in wasted HIV-infected patients starting or on ART is therefore common, because their elevated nutritional needs are often challenging to meet in resource-constrained settings [10].

In developing countries, paediatric formulas of lipid-based ready-to-use foods energy-dense spreads - have also been used for the treatment of malnutrition among wasted HIV-infected adults with some beneficial effects on weight gain [11-12]. However, poor acceptability of lipid-based nutrient supplements (LNS) has been reported among HIV-infected adults and monotony of the diet was one of the main limiting factors to the LNS acceptability in adults [13]. In addition, chemosensory changes associated with HIV infection or ART may also influence the acceptability of LNS [14-17]. New flavours of LNS with high organoleptic acceptability for HIVinfected adults are therefore urgently needed. Indeed, LNS formulations more suited to HIV-infected adults' taste are thought to increase their compliance to these supplements [13].

Nutriset SAS (Malaunay, France) has developed new formulations and flavours of LNS targeting undernourished HIV-infected adults to be used in the Nutritional Support for African adults Starting Antiretroviral Therapy (NUSTART) trial (Pan African Clinical Trials Registry; registration \# PACTR201106000300631). In preparation for this trial, the present sensory evaluation was conducted to assess the acceptability of the new LNS flavours among HIV-infected adults in Tanzania. The objectives of the study were to (i) determine the acceptability levels of the different LNS formulations (ii) understand and describe the sensory attributes of the formulations and (iii) establish the mode of consumption driving the acceptability levels of the formulations in this population.

\section{MATERIAL AND METHODS}

\section{Study area and participants}

The acceptability trial was conducted in the HIV clinic of the Nyamagana District Hospital, Mwanza region, Tanzania from July to August 2011. Convenience sampling 


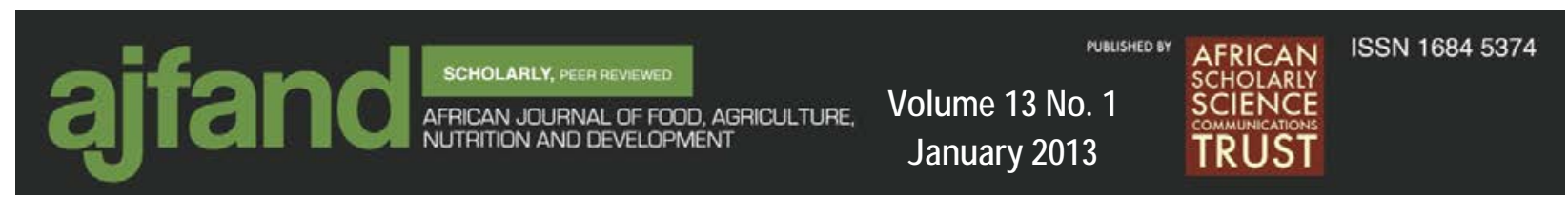

was used to recruit the participants. Eligible participants were adults aged 18 and above, infected with HIV, and either already on ART or now meeting ART eligibility criteria according to the Tanzanian National AIDS Control Program at the time. The ART eligibility criteria in Tanzania are CD4 counts $\leq 200$ cells $/ \mathrm{mm}^{3}$ or WHO stage III or IV and CD4 counts $\leq 350$ cells $/ \mathrm{mm}^{3}$ [18]. Since these were previously untested products, pregnant or lactating women were excluded. For logistic reasons we also excluded people unable to eat or who were involved in another potentially confounding trial. Eligible subjects who provided written consent were invited to undertake a single session of sensory test.

Upon recruitment, participants' weight and height were measured to the nearest $0.1 \mathrm{~kg}$ and $0.1 \mathrm{~cm}$ respectively. Information on sex, age, smoking, hunger and appetite, changes in taste, current medication, most recent CD4 count, last 24 hours morbidity, time of last meal and when ART was started was obtained from the participants.

This study was a pilot for the NUSTART trial and was approved by the Ethical Committee of London School of Hygiene and Tropical Medicine, London, United Kingdom and the National Institute for Medical Research, Dar es Salaam, Tanzania.

\section{LNS products}

The supplements were formulated and manufactured by Nutriset SAS (Malaunay, France). They were packaged in $50 \mathrm{~g}$ sachets and specifically labelled for the study. Two types of LNS were developed in preparation for the NUSTART trial: LNS-30 g for pre-ART nutritional support and LNS-250 g for nutritional support during ART. LNS-30 g has a high nutrient density, except iron, with a daily dose of $30 \mathrm{~g}$. LNS-250 $\mathrm{g}$ has a lower nutrient density but contains iron and $250 \mathrm{~g}$ are to be consumed per day. The common ingredients of the different LNS formulations were vegetable oil, peanuts, maize starch and soy flour, fortified with vitamins and minerals. For each type of LNS, there were "sweet" formulations with three different flavours (Chocolate, Chocolate-Banana and Unflavoured) and "salty or non-sweet" formulations also with three different flavours (Tomato, Tomato-Onion and Unflavoured). Therefore, there were four different sets of LNS for the study (Table 1).

The acceptability of all the formulations was investigated both raw and mixed with local foods. The sweet formulations were mixed with a local maize-based porridge while the non-sweet formulations were associated with stiff maize porridge "ugali" plus green-leafy vegetables "mchicha". The porridge mixture was prepared by mixing $45 \mathrm{~g}$ of the sweet formulation with $50 \mathrm{~g}$ of fresh local maize-based porridge. The same serving size has previously been used in sensory trial involving porridge mixed with LNS $[18,19]$. The tested meal including the non-sweet formulation was composed of approximately $15 \mathrm{~g}$ ugali, $5 \mathrm{~g}$ of mchicha and $10 \mathrm{~g}$ of LNS for a total serving size of approximately $30 \mathrm{~g}$ which was enough for several tastings. The foods were freshly prepared every morning following a local recipe and carried to the study site in a thermos flask and pot. 


\section{Sensory trial protocol}

Block randomisation was used to assign the four sets of supplements to the participants. The products were assigned a three-digit identification number generated using Excel (Microsoft, Redmond, USA) and their identity was concealed from the participants and the interviewer. The participants were asked to have one spoonful or one bite of the product for the subsequent tests, as described below. Drinking water at room temperature was available for each participant.

\section{Descriptive test}

Hedonic rating scales were used to assess the sensory attributes potentially associated with complaints and to determine if the new formulations and flavours were appreciated by the participants. After the first spoon or bite, the participants were asked to identify and list the ingredients used for the formulation of the supplements. They were asked to assess the intensity of sugar, salt, oil, smell, thickness using a Just-about-right scale ( 1 = Not at all enough, $5=$ Too much); and how pleasant was the taste of the formulations using a 5 -point hedonic scale $(1=$ Very bad, $5=$ Very good).

\section{Hedonic test}

Participants were asked to indicate their overall liking of the product by rating how much they like or dislike the products using a 5-point hedonic scale ( 1 = Dislike a lot, $5=$ Like a lot) and then select the main attributes driving their liking or disliking of the products from a list presented to them.

\section{Food-Action Rating test (FACT)}

As a proxy for the acceptability of the products, participants were asked to choose among the following five sentences the one which best describes their willingness to consume the food if freely available:

1. I extremely like this product and I would eat it at every opportunity

2. I moderately like this product and I would eat it very often

3. I neither like or dislike this product and I would eat it every now and then

4. I moderately dislike this product but I would eat it occasionally

5. I extremely dislike this product and I would never eat it

\section{Preference test}

The participants were then asked to taste the whole set of products for a second time and to rank the products in order of preference with the best as number 1 and the worst as number 3. Finally, they were given the opportunity to make additional comments on the products.

\section{Statistical analysis}

Data were entered into an Excel database (Microsoft, Redmond, USA) and then, analysed with STATA version 11.2 (StataCorp, College Station, USA). Quantitative data for normally distributed variables, as assessed by box plots and skewness, were described using mean, standard deviation (SD) and proportion; and compared using Fisher's exact test. Non-normally distributed variables were described using median and range or proportion. Responses from 5-point rating scales were treated as ordinal 


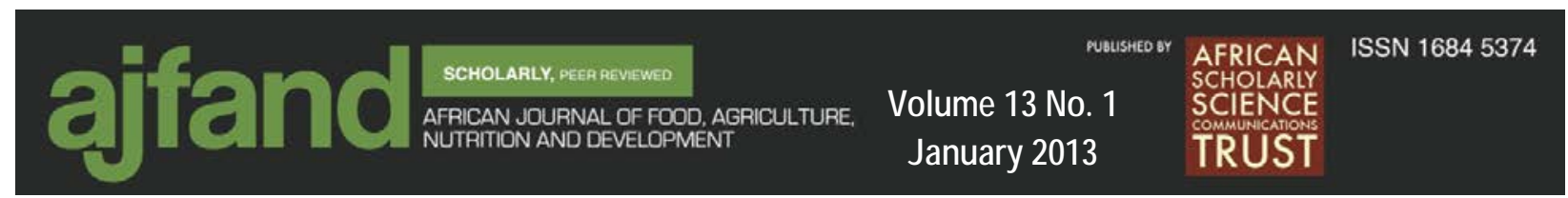

data and analyzed using non-parametric tests: Kruskal-Wallis test, Wilcoxon signedrank test or Wilcoxon sum rank test to compare 2 groups and Friedman test to compare more than 2 groups, as appropriate [11]. P-value $<0.05$ was considered significant for all comparisons. The answers to the open-ended questions of the descriptive test were coded by reported ingredients and then analysed using proportions. Spearman's correlation coefficients were calculated to investigate the associations of the sensory attributes with overall degree of liking (DOL).

\section{RESULTS}

\section{Participants and background characteristics}

Forty participants were recruited, ten for each set of LNS. One did not take part in the testing of LNS mixed with food because she did not like the local food used in mixing the LNS. Trial participants in the four LNS groups were found to be similar so characteristics are shown combined (Table 2). The median age of the participants was 39 years; most of them were female and of normal BMI. The majority were on ART (95\%) and 93\% were taking cotrimoxazole, (antimicrobial for opportunistic infections prophylaxis). The most common reported morbidity symptoms or discomforts in the past 24 hours were cold (43\%), fever (35\%) and cough (33\%). Most participants reported being hungry before the sensory trial. Appetite and taste changes were common in the studied population and none of the participants were smokers.

\section{LNS acceptability and preference according to the type of product}

All the sets of products regardless of the formulations and the flavours were positively evaluated. As shown in Table 3, all the investigated attributes i.e. sugar, salt, oil, thickness, and smell were perceived "just about right". The taste received a median rate of 4-Good. All the sets received median DOL scores of either 4-I like it a little or 5-I like it a lot and median FACT scores of 2-I would eat it very often. When comparing the sets of products, the set of LNS-30 g non-sweet (tomato, tomato-onion and unflavoured) had the lowest median DOL of 4-I like it a little compared to all the other sets which each had a median DOL of 5-I like it a lot $(\mathrm{p}=0.0005)$. Despite the fact that the set of LNS-30 g non-sweet was less appreciated, there was no significant difference in terms of FACT rating and preference compared to the other sets.

\section{Sweet formulations}

For both $30 \mathrm{~g}$ and $250 \mathrm{~g}$ sweet LNS served without food, there were no overall significant differences in organoleptic properties or acceptability of the different formulations (chocolate, chocolate-banana, unflavoured). All LNS-30 g sweet formulations were considered acceptable for with a median score of 3-Just about right for the intensity of the studied organoleptic characteristics while the taste received a median score of 4-Good and the median overall DOL was 5-Like a lot for each flavour. Regarding the prospective consumption pattern, all these flavours received a favourable median FACT rate of 2-I will eat it very often. Comparable results for 250 g products are shown in Figure 1. 


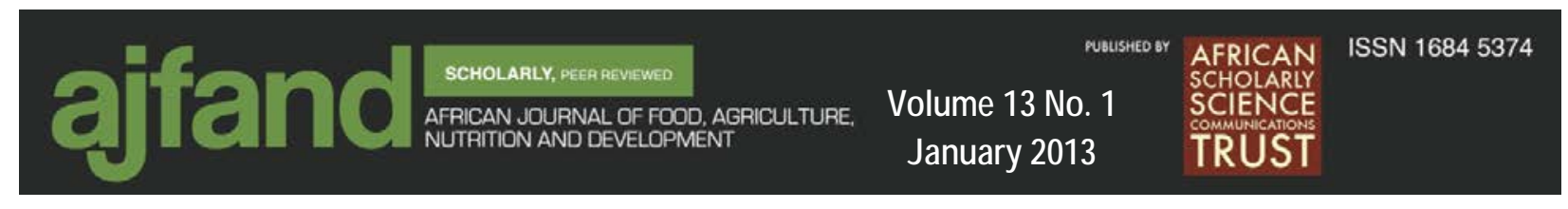

Acceptability of LNS-250g sweet formulation according to flavours

*Chocolate $(\mathrm{n}=10) \quad-$ Chocolate-Banana $(\mathrm{n}=10) \rightarrow$ Unflavoured sweet $(\mathrm{n}=10)$

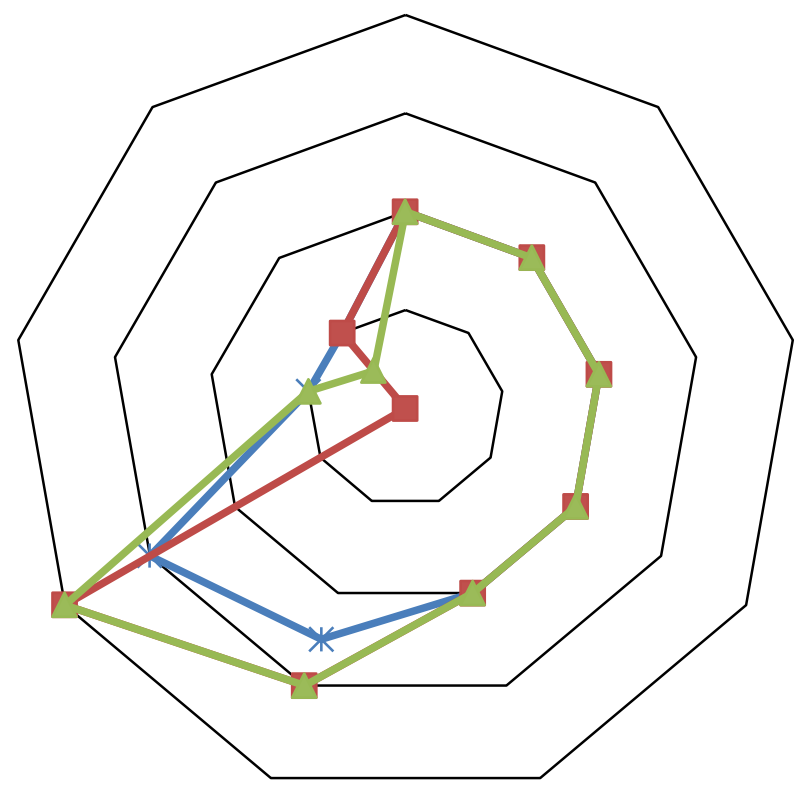

Figure 1: Acceptability of different flavours of LNS-250 g sweet formulations

Note that for the three flavours the values for the intensity of sugar, salt, oil, thickness and smell are completely overlapped.

\section{Non-sweet formulations}

The tomato, tomato-onion and unflavoured formulations of LNS-30 g were found acceptable with a median of 3-Just about right for the organoleptic attributes; and a median rate of 4-Good for the taste (Figure 2). There was no significant difference in terms of overall DOL $\quad(\mathrm{p}=0.60)$, with all the flavours rated 4-I like it a little. The tomato flavour was ranked as the best, following by the tomato-onion and the unflavoured non-sweet formulation but the difference was not significant ( $p=0.18$ ). Similarly, the tomato, tomato-onion and unflavoured formulations of LNS-250 g were found acceptable in term of organoleptic attributes, FACT rate and preference and there was no significant different between these three products. However, the three flavours of LNS-250 g non-sweet formulation received a DOL rank of 5-I like it a lot and were significantly more appreciated than the non-sweet formulations of LNS-30 g $(p=0.004)$. 


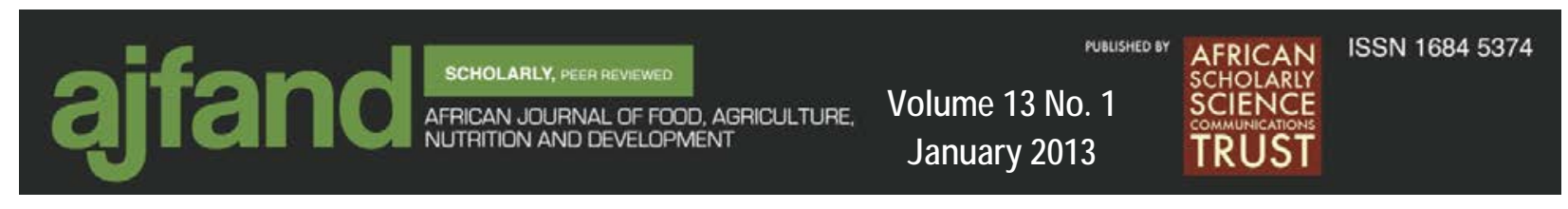

\section{Acceptability of LNS-30g non sweet formulation according flavours}

$\leadsto$ Tomato $\rightarrow$ Tomato-Onion $\rightarrow$ Unflavoured salty

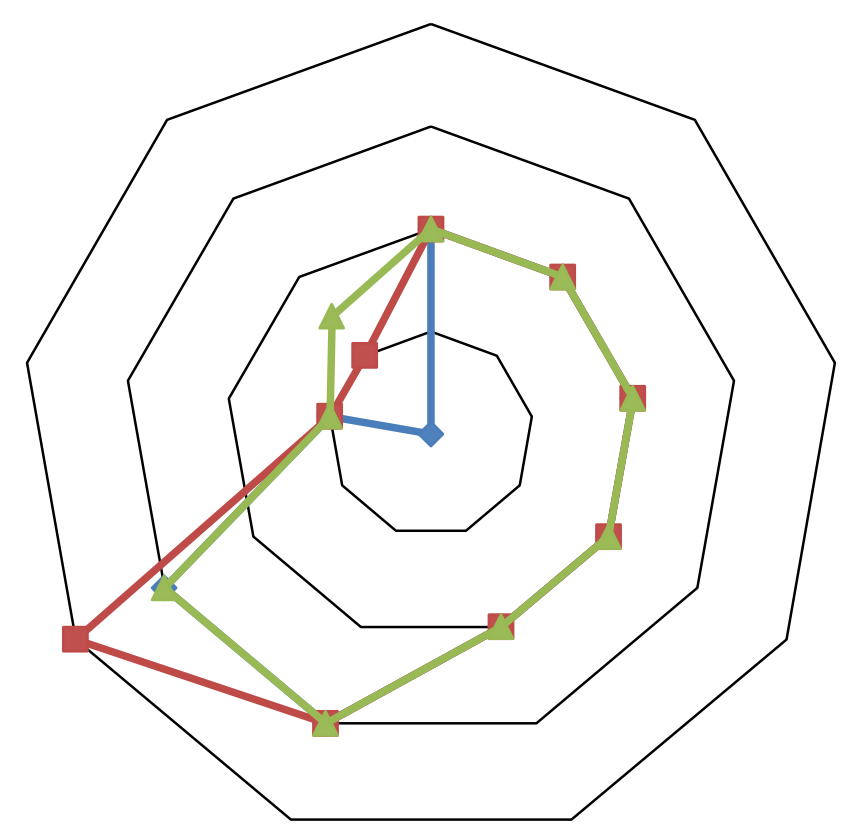

Figure 2: Acceptability of different flavours of LNS-30 g non sweet formulations

Note that for the three flavours the values for the intensity of sugar, salt, oil, thickness and smell as well as for the taste are completely overlapped.

\section{Effect of the association with local food on LNS acceptability}

All the LNS associated with foods were considered acceptable by the participants. Indeed, all the organoleptic characteristics received a median rate of 3-Just about right and 4-Good for the taste. The median overall DOL and FACT rates were respectively 5-Like a lot and 2-Very often. When comparing these two modes of consumption for LNS-30 g, there was no significant difference regarding the overall DOL (Table 4), the FACT rate and the preference, regardless of the flavour. These results were similar for LNS-250 g.

\section{Organoleptic characteristics influencing LNS acceptability}

Most of the participants were familiar with some of the LNS ingredients. Indeed, in the sweet formulations, 65\% of the participants accurately recognised groundnut and $33 \%$ banana. In the non-sweet formulations, 99\% accurately identified groundnut and $15 \%$ tomato. Among the studied sensory attributes, the taste, followed by the smell, the intensity of sugar and salty taste were the most cited by the participants liking LNS-30 g sweet formulations (chocolate, chocolate-banana and unflavoured). 
Regarding LNS-30 g non-sweet formulations (tomato, tomato-onion and unflavoured), the taste and the intensity of the smell were the organoleptic characteristics most frequently cited by the participants as the main attributes explaining their liking. Similar results were found for LNS-250 g. No participants disliked the non-sweet formulations and only two expressed dislike of the sweet formulations, one because of the taste and the other because of the salty taste.

The investigation of the organoleptic characteristics driving the overall acceptability of the supplements revealed that only the intensity of sugar and the taste were significantly and positively correlated with the overall DOL in the LNS-30 g without food (Table 5). The thickness was negatively correlated with the FACT rates (coefficient: -0.27; $\mathrm{p}=0.034$ ). However, none of the sensory attributes were significantly correlated with the preference. In LNS-250 g, only the intensity of salt was significantly correlated with the DOL (coefficient: $0.28 ; \mathrm{p}=0.027$ ). The smell was negatively correlated with the FACT rates (coefficient: $-0.27 ; \mathrm{p}=0.043$ ). There were no significant correlations between the studied attributes and the preference.

\section{DISCUSSION}

The acceptability of new flavours of LNS was investigated among HIV-infected Tanzanian adults. All the formulations of LNS were well accepted by the studied population. Indeed, all the studied sensory attributes and the overall DOL were reported acceptable and the FACT rates predicted frequent consumption of these supplements. The set of LNS-30 g non-sweet was the least appreciated compared to the other sets which were equally appreciated (LNS-30 g sweet, LNS-250 g sweet and LNS-250 g non-sweet). The thickness in LNS-30 g was negatively correlated with the FACT rates. Thickness as a limiting factor to LNS acceptability was previously suggested in a study involving HIV-infected adults [13]. The association with local foods did not influence either positively or negatively the acceptability of these products. The taste, the smell, the intensity of sugar and salt were the most important factors contributing to the good acceptability of the products.

Before starting the sensory trial, almost all the participants reported being hungry and hunger is known to influence food acceptability and preference. Furthermore, taste preference for sugar and fats may be universal and innate [19]. This might explain why the LNS-30 g sweet formulations were more appreciated than the non-sweet ones. The high acceptability of all the supplements may also be explained by people's previous experience with some of the supplements' ingredients. Indeed, it has been shown that a previous experience with a food can contribute to its increased acceptability [20]. In this study, most of the participants were familiar with the flavours of the supplements.

The sensory trial was conducted at the HIV clinic of Nyamagana district hospital. Although interviews were conducted in a particular room allocated for the research project, the HIV clinic environment might have influenced people's perceptions. Food acceptability is influenced by the context in which food is evaluated [21] and a private environment was created to reproduce the usual environment of food eaten outside the 


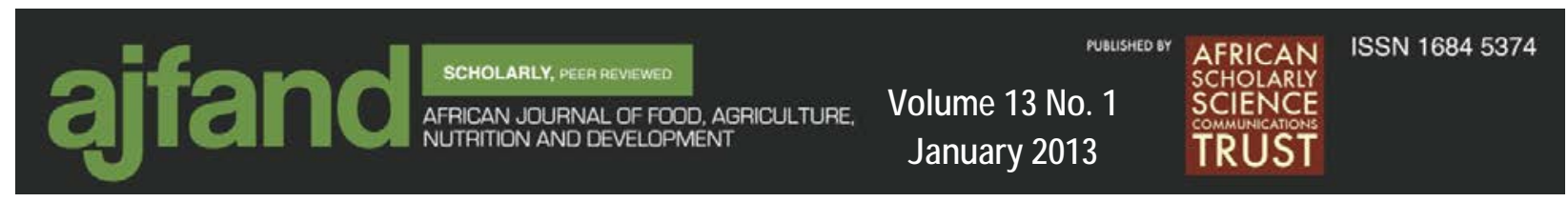

home. In adults, contrary to children, taste preferences and aversions are not always direct predictors of food consumption. Consequently, it is difficult to link adult taste preferences with intake measurements, whether in a laboratory setting or in real life [17]. Moreover, as few studies have investigated taste response, food preference and intake patterns in the same adult population, the link between taste and food consumption is often indirectly established. Therefore, the results of the descriptive test were combined with the FACT rating and preference tests in order to evaluate the acceptability of the tested products. The triangulation of all these tests provided coherent results. All the rating scales used in this study were standardised scales previously used in resource-constrained settings among children and adults for the evaluation of the acceptability of different types of foods [22; 24]. In the present study, at least $90 \%$ of the panellists in each sensory group reported liking the supplements. Thus, the different new flavours may help to prevent monotony when LNS are consumed during a longer period. Nevertheless, it is uncertain whether appreciation and consumption pattern change when the products are consumed for a longer time. More investigation is needed to study the effect of time on the appreciation and the consumption pattern of these new flavours of LNS among HIVinfected adults.

The main limitation of this study was that sample size was low for logistic reasons. Furthermore, few participants were undernourished and most of them had a relatively good health status as a result of their access to ART; the median time since starting ART was 17.5 months. Whether the same results will be obtained in wasted HIVinfected adults is uncertain since they might suffer severe clinical conditions affecting their ability to swallow or their chemosensory functions.

\section{CONCLUSION}

The high early mortality rate among undernourished adults infected with HIV and the high acceptability of the tested LNS flavours point towards the need to assess the effectiveness of these supplements in HIV-infected adults. As the flavours were well accepted, all of them can be used in effectiveness trials, providing choice and variety to HIV-infected adults consuming them for prolonged periods. Further research on the acceptability of these supplements over the longer term and in settings where food insecurity and dietary patterns are different is warranted.

\section{ACKNOWLEDGEMENTS}

The work was conducted in partial fulfilment of the requirements for Marlene Hebie's MSc degree at the London School of Hygiene and Tropical Medicine. We acknowledge the assistance of Mette Olsen and Daniela Manno in the design of the sensorial trial. We thank Rose-Mary Kolela and David Mandili for assistance with field work. We kindly thank the staff of Nyamagana District Hospital where the study was carried out as well as the study participants. Finally, our thanks go to Mamane Zeilani and Marine Le Chatelier of the external research and nutrition strategies department of Nutriset SAS for their support regarding the development and shipment of the nutritional supplements. 


\section{Source of funding}

Funding for the study was from Nutriset SAS, Malaunay, France.

\section{Conflicts of interest}

At the time of the study, Svenja Jungjohann was employed as a Nutrition Research Leader by Nutriset SAS that produced the supplements. The remaining authors have declared no conflicts of interest. 
Table 1: Composition of the four sets of LNS used in the sensory trial

\begin{tabular}{llll}
\hline SET & Type of LNS & Formulation & Flavours \\
\hline $\mathbf{1}$ & LNS-30 g & Sweet & Chocolate \\
& & Chocolate-Banana \\
& & Unflavoured \\
$\mathbf{2}$ & LNS-30 g & Non-sweet & Tomato \\
& & & Tomato-Onion \\
& & & Unflavoured \\
$\mathbf{3}$ & LSN-250 g & Sweet & Chocolate \\
& & & Chocolate-Banana \\
& & & Unflavoured \\
$\mathbf{4}$ & LNS-250 g & Non-sweet & Tomato \\
& & & Tomato-Onion \\
& & & Unflavoured \\
\hline
\end{tabular}


Table 2: Characteristics of the participants ${ }^{1}$

\section{Characteristics}

Age, Median (range), years

Female, No (\%)

BMI, Median (range), $\mathrm{Kg} / \mathrm{m}^{2}$

BMI categories [No (\%), $\left.\mathbf{k g} / \mathrm{m}^{2}\right]$

\section{Participants}

39 (26-70)

$30(75 \%)$

$21.1(16.6-35.9)$

\begin{tabular}{ll}
$<16.9$ & $1(3 \%)$ \\
$17.0-18.4$ & $2(5 \%)$ \\
$>18.5$ & $37(92 \%)$ \\
\hline CD4 count, Median (range), cells/mm $\mathrm{mm}^{3}$ & $192(14-645$ \\
Current medication, No (\%) & \\
\hline ART $^{2}$ & $37(93 \%)$ \\
Septrin & $38(95 \%)$ \\
Anti-Tuberculosis & $1(3 \%)$ \\
Other medication & $4(10 \%)$
\end{tabular}

Morbidity/discomfort in the past 24 hours, No (\%)

Nausea

$9(23 \%)$

Changes in taste

9 (23\%)

Cold 17 (43\%)

Cough

$13(33 \%)$

Pain when swallowing $4(10 \%)$

Fever

$14(35 \%)$

Hungry $32(80 \%)$

\section{Appetite, No (\%)}

Excellent

$1(3 \%)$

Good $25(62 \%)$

Average $7(17 \%)$

Poor $6(15 \%)$

Very poor $1(3 \%)$

Taste, No (\%)

Very good 0

Good 25 (62\%)

Average $8(20 \%)$

Bad $5(13 \%)$

Very bad $2(5 \%)$

${ }^{1}$ Sample size was 40 except for CD4 count which was available for only 36 participants.

${ }^{2}$ Median time on ART was $17.5(1-71)$ months 
Table 3: Acceptability of the 4 sets of LNS without food

\begin{tabular}{|c|c|c|c|c|c|}
\hline Attributes & $\begin{array}{l}30 \text { g sweet } \\
(n=10)\end{array}$ & $\begin{array}{l}30 \text { g non-sweet } \\
(n=10)\end{array}$ & $\begin{array}{l}250 \mathrm{~g} \text { sweet } \\
(n=10)\end{array}$ & $\begin{array}{l}250 \text { g non-sweet } \\
(n=10)\end{array}$ & p-value ${ }^{1}$ \\
\hline Sweet & & & & & 0.0001 \\
\hline Median (range) & $3(1-4)$ & $3(1-3)$ & $3(3-4)$ & $3(2-3)$ & \\
\hline Considered acceptable, $\%^{2}$ & $80 \%$ & $60 \%$ & $90 \%$ & $90 \%$ & \\
\hline Salt & & & & & 0.42 \\
\hline Median (range) & $3(2-4)$ & $3(2-4)$ & $3(2-3)$ & $3(2-4)$ & \\
\hline Considered acceptable, $\%$ & $90 \%$ & $90 \%$ & $95 \%$ & $95 \%$ & \\
\hline Oil & & & & & 0.18 \\
\hline Median (range) & $3(3-4)$ & $3(2-3)$ & $3(3-4)$ & $3(3-4)$ & \\
\hline Considered acceptable, $\%$ & $96 \%$ & $96 \%$ & $95 \%$ & $97 \%$ & \\
\hline Thickness & & & & & 0.0002 \\
\hline Median (range) & $3(1-5)$ & $3(3-5)$ & $3(3-4)$ & $3(3-5)$ & \\
\hline Considered acceptable, $\%$ & $90 \%$ & $80 \%$ & $95 \%$ & $70 \%$ & \\
\hline Smell & & & & & 0.18 \\
\hline Median (range) & $3(3-5)$ & $3(3-3)$ & $3(3-4)$ & $3(3-5)$ & \\
\hline Considered acceptable, $\%$ & $80 \%$ & $100 \%$ & $85 \%$ & $85 \%$ & \\
\hline Taste & & & & & 0.92 \\
\hline Median (range) & $4(1-5)$ & $4(3-5)$ & $4(2-5)$ & $4(1-5)$ & \\
\hline Considered acceptable, $\%$ & $90 \%$ & $80 \%$ & $80 \%$ & $80 \%$ & \\
\hline Overall DOL³ , Median (range) & $5(1-5)$ & $4(4-5)$ & $5(4-5)$ & $5(2-5)$ & 0.0005 \\
\hline Food-Action-Rating, Median(range) & $2(1-5)$ & $2(1-3)$ & $2(1-3)$ & $1(1-4)$ & 0.62 \\
\hline Preference & $2(1-3)$ & $2(1-3)$ & $2(1-3)$ & $2(1-3)$ & 1.0 \\
\hline
\end{tabular}

${ }^{1}$ Kruskal-Wallis test

${ }^{2}$ Percentage of products found to be acceptable: "Just about right" for the intensity of sugar, salt, oil, smell and the thickness; "Good" and "Very good" for the taste

${ }^{3}$ Degree of liking 
Table 4: Influence of consuming with food on the acceptability of LNS-30 g

\begin{tabular}{llll}
\hline DoL, Median (range) & $\begin{array}{l}\text { Without food } \\
(n=10)\end{array}$ & $\begin{array}{l}\text { With } \\
(n=10)\end{array}$ & Food $^{\text {p-value }}$ \\
\hline Chocolate & $5(4-5)$ & $5(4-5)$ & 0.58 \\
Chocolate-banana & $5(1-5)$ & $5(4-5)$ & 0.61 \\
Unflavoured sweet & $5(1-5)$ & $5(4-5)$ & 0.56 \\
Tomato & $4(4-5)$ & $4.5(4-5)$ & 0.18 \\
Tomato-Onion & $4(4-5)$ & $4(4-5)$ & 0.56 \\
Unflavoured non-sweet & $4(4-5)$ & $4(4-5)$ & 0.08 \\
\hline
\end{tabular}

${ }^{1}$ Wilcoxon sign rank sum test 
Table 5: Correlation between the sensory attributes and overall $\mathrm{DOL}^{1}$ of raw LNS-30 $g$ and LNS-250 g

\begin{tabular}{lllll}
\hline \multirow{2}{*}{ Attributes } & \multicolumn{2}{l}{ LNS-30 g DOL } & \multicolumn{2}{l}{ LNS-250 g DOL } \\
\cline { 2 - 5 } & $\begin{array}{l}\text { Correlation } \\
\text { coefficient }(\boldsymbol{\rho})^{2}\end{array}$ & p-value & $\begin{array}{l}\text { Correlation } \\
\text { coefficient }(\boldsymbol{\rho})^{2}\end{array}$ & p-value \\
\hline Sugar & 0.35 & 0.006 & 0.07 & 0.60 \\
Salt & 0.10 & 0.46 & 0.29 & 0.027 \\
Oil & 0.00001 & 1.00 & 0.15 & 0.25 \\
Taste & 0.29 & 0.025 & 0.23 & 0.08 \\
Thick & -0.06 & 0.63 & -0.17 & 0.20 \\
Smell & -0.01 & 0.94 & 0.11 & 0.41 \\
\hline
\end{tabular}

${ }^{1}$ Degree of liking

${ }^{2}$ Spearman's rank correlation coefficient 


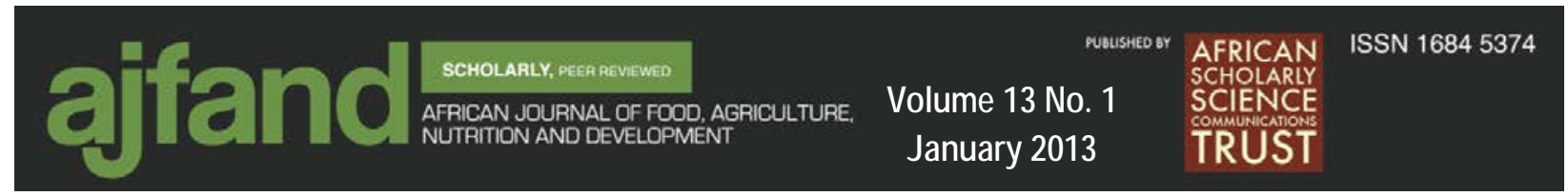

\section{REFERENCES}

1. DHS. Tanzania HIV/AIDS and Malaria Indicator Survey 2008 (THMIS). http://www.measuredhs.com/pubs/pdf/AIS6/AIS6_05_14_09.pdf Accessed: 08 July 2011.

2. Koethe JR and DC Heimburger Nutritional aspects of HIV-wasting syndrome in Sub-Saharan Africa. Am J Clin Nutr. 2010; 91(suppl):1138S$1142 S$.

3. Lawn SD, Harries AD, Anglarete X, Myerg L and R Wood Early mortality among adults accessing antiretroviral treatment programmes in sub-Saharan Africa. AIDS. 2008; 22:1897-1908.

4. Koethe JR, Chi BH, Megazzini KM, Heimburger DC and JSA Stringer Macronutrient Supplementation for Malnourished HIV-Infected Adults: A Review of the Evidence in Resource-Adequate and Resource-Constrained Settings. Clin Infect Dis. 2009; 49:787-798.

5. Moore DM, Yiannoutsos CT, Musick BS, Tappero J, Degerman R, Campbell J, Were W, Kaharuza F, Alexander LN, Downing $R$ and $J$ Mermin Determinants of early and late mortality among HIV-infected individuals receiving home-based antiretroviral therapy in rural Uganda. $J$ Acquir Immune Defic Syndr. 2011; 58(3):289-296.

6. Moh R, Danel C, Messou E, Ouassa T, Gabillard D, Anzian A, Abo Y, Salamon R, Bissagnene E, Seyler C, Eholié S and X Anglaret Incidence and determinants of mortality and morbidity following early antiretroviral therapy initiation in HIV-infected adults in West Africa. AIDS. 2007; 21:2483-2491.

7. Etard JF, Ndiaye I, Thierry-Mieg M, Guèye NF, Guèye PM, Lanièce I, Dieng AB, Diouf A, Laurent C, Mboup S, Sow PS and E Delaporte Mortality and causes of death in adults receiving highly active antiretroviral therapy in Senegal: a 7-year cohort study. AIDS. 2006; 20:1181-1189.

8. Zachariah R, Fitzgerald M, Massaquoi M, Pasulani O, Arnould L, Makombe S and AD Harries Risk factors for high early mortality in patients on antiretroviral treatment in a rural district of Malawi. AIDS. 2006; 20:23552360 .

9. WHO. Nutrient requirements for people living with HIV/AIDS: report of a technical consultation. Geneva: WHO; 2003. 
10. Cantrell RA, Sinkala M, Megazinni K, Lawson-Marriott S, Washington S, Chi BH, Tambatamba-Chapula B, Levy J, Stringer EM, Mulenga L and JS Stringer A pilot study of food supplementation to improve adherence to antiretroviral therapy among food-insecure adults in Lusaka, Zambia. $J$ Acquir Immune Defic Syndr. 2008; 49:190-195.

11. Bahwere $\mathbf{P}$, Sadler $\mathbf{K}$ and $\mathbf{S}$ Collins Acceptability and effectiveness of chickpea sesame-based ready-to-use therapeutic food in malnourished HIVpositive adults. Patient Prefer Adherence. 2009; 3:67-75.

12. Ndekha MJ, Van Oosterhout JJ, Zijlstra EE, Manary M, Saloojee $\mathbf{H}$ and MJ Manary Supplementary feeding with either ready-to-use fortified spread or corn-soy blend in wasted adults starting antiretroviral therapy in Malawi: randomised, investigator blinded, controlled trial. BMJ. 2009; 338:b1867. doi: 10.1136/bmj.b1867.

13. Dibari F, Bahwere P, Le Gall I, Guerrero S, Mwaniki D and A Seal A qualitative investigation of adherence to nutritional therapy in malnourished adult AIDS patients in Kenya. Public Health Nutr. 2011. doi:10.1017/S1368980010003435.

14. Johnson MO, Stallworth T and TB Neilands The Drugs or the Disease? Causal Attributions of Symptoms Held by HIV-Positive Adults on HAART. AIDS and Behavior. 2003; 7(1):109-117.

15. Heald AE, Pieper CF and SS Schiffman Taste and smell complaints in HIVinfected patients. AIDS. 1998; 12:1667-1674.

16. Graham CS, Graham BG, Bartlett JA, Heald AE and SS Schiffman Taste and smell losses in HIV infected patients. Physiol Behav. 1995; 58(2):287293.

17. Drewnowski A Taste preference and food intake. Annu Rev Nutr. 1997; 17:237-253.

18. NACP National Guidelines for the management of HIV and AIDS. Third edition. Ministry of Health and Social Welfare, Government of Tanzania, Dares-Salam. 2009.

19. Drewnowski A and C Hann Food preferences and reported frequencies of food consumption as predictors of current diet in young women. Am J Clin Nutr. 1999; 70:28-36.

20. Rozin P and TA Vollmecke Food likes and dislikes. Ann Rev Nutr. 1986; 6:433-456. 
21. Hess SY, Bado L, Aaron GJ, Ouedraogo JB, Zeilani M and KH Brown Acceptability of zinc-fortified, lipid-based nutrient supplements (LNS) prepared for young children in Burkina Faso. Matern Child Nutr. 2011. doi: 10.1111/j.1740-8709.2010.00287.x

22. Adu-Afarwuah S, Lartey A, Zeilani M and KG Dewey Acceptability of lipid-based nutrient supplements (LNS) among Ghanaian infants and pregnant or lactating women. Matern Child Nutr. 2010. doi: 10.1111/j.17408709.2010.00286.x

23. Owino VO, Sinkala M, Amadi B, Tomkins AM and SM Filteau Acceptability, storage stability and costing of $\alpha$-amylase-treated maize-beansgroundnuts-bambaranuts complementary blend. J Sci Food Agric. 2007; 87:1021-1029. 